\title{
MECANISMOS DE PATOGÉNESIS BACTERIANA EN PROCESOS RESPIRATORIOS: PASTEURELLOSIS
}

\section{MECHANISMS OF BACTERIAL PATHOGENESIS IN RESPIRATORY PROCESSES: PASTEURELLOSIS}

\author{
Paula Esquinas Castillo ${ }^{1}$ \\ Carlos Iregui Castro ${ }^{2}$
}

\author{
RESUMEN
}

Pasteurella multocida es un patógeno multiespecies que causa serias enfermedades en animales de producción, animales silvestres, de laboratorio y en los seres humanos. Esta bacteria es el agente etiológico de enfermedades, como el cólera aviar en pollos y pavos, septicemia hemorrágica en bovinos, rinitis atrófica en cerdos e infecciones en humanos por mordeduras de gatos y de perros. Este agente es responsable por pérdidas anuales de cientos de millones de dólares en producción animal solo en Estados Unidos. En los conejos P. multocida puede ser un patógeno virulento induciendo cuadros desde septicemia fatal, neumonía, rinitis crónica, otitis media, así como abscesos diseminados. En esta especie, se presenta un síndrome respiratorio, en el cual, frecuentemente,, se aísla también Bortetella bronchiseptica, sin embargo aun no se ha determinado el papel que desempeña cada una de estas bacterias o algunos de sus factores de virulencia como el LPS en la inducción de la enfermedad. P. multocida es un cocobacilo Gram negativo donde se han identificado varios factores de virulencia, como la cápsula, la sialidasa, la hemagluti-

${ }^{1}$ MV, M.Sc.(c), Grupo de Investigación en Patobiología Veterinaria, Maestría en Salud y Producción Animal, Universidad Nacional de Colombia, Ciudad Universitaria, pcesquinasc@unal.edu.co, Bogotá, Colombia.

2 MV. DMV. Director Grupo de Investigación en Patobiología Veterinaria, Departamento de Patología, Universidad Nacional de Colombia, Ciudad Universitaria, caireguic@unal.edu.co, Bogotá, Colombia. nina filamentosa, la fimbria, la neuraminidasa, algunas porínas, la toxina dermonecrótica, la toxina termolabil (PMT), las proteínas de membrana externa (OMP) y el LPS. La falta de prevención eficaz y control de esta clase de enfermedades hace necesario la investigación de la patogénesis de este tipo de bacterias y sus interacciones con el hospedero.

Palabras clave: Pasteurella multocida, patogénesis, factores de virulencia, lipopolisacárido.

\section{SUMMARY}

Pasteurella multocida is a multispecies pathogen that causes serious diseases in wild and laboratory animals and in human beings. This bacteria is the etiologic agent of diseases such as fowl cholera in chicken and turkey, hemorrhagic septicemia in cattle, athrofic rhinitis in swine and infections in humans by cat and dog bites. This agent is responsible for annual losses of hundreds of millions of dollars in animal production, only in United States. In the rabbit $P$. multocida can be a virulent fatal pathogen inducing septicemia, pneumonia, chronic rhinitis, otitis as well as abscesses. In this species a respiratory syndrome appears in which frequently Bortetella bronchiseptica is also isolated. However the role of each of these bacteria or some of their virulence factors, like LPS, in the induction of the disease has not been determined. P. multocida is a Gram negative cocobacilo with several identified virulence factors, like capsule, sialidasa, filamentous hemagglutinin, flagelum, neuraminidasa, some 
porins, dermonecrotic toxin, termolabil toxin (PMT), outer membrane proteins (OMP) and LPS. The lack of effective prevention and control of this type of diseases makes a research of the pathogenesis of the bacteria and its interactions with the host necessary.

Key words: Pasteurella multocida, pathogenesis, virulence factors, lipopolysaccharide.

\section{INTRODUCCIÓN}

En general, las patologías inducidas por bacterias Gram negativas tienen un impacto muy importante sobre los animales de producción sometidos a altas densidades y por ende a mala calidad del ambiente (Meyer, 1991; Bonilla \& García-Delgado, 1993; Murtaugh et al. 1996). Dentro de estas enfermedades, las entidades respiratorias son consideradas como causantes de grandes pérdidas económicas en distintas especies animales (Andrews E Kennedy, 1997; Bureau et al. 2001). En bovinos, aves y cerdos se han demostrado pérdidas anuales de millones de dólares tanto por muertes como por bajas en la producción (Coy et al. 1997; Griffin, 1997; Brockmeier \& Register, 2000). En conejos, reiteradamente, se muestra una estrecha asociación entre Pasteurella multocida con diversas patologías (Flatt $\mathcal{E}$ Dungworth, 1971a y b; DiGiacomo et al. 1983; Deeb et al. 1990; Kpodekon et al. 1999). Si bien todas las formas de presentación reportadas tienen algún grado de significancia económica, son las formas respiratorias (rinitis y neumonía) y la forma sistémica (septicemia) las de mayor impacto negativo en los planteles dedicados a la explotación comercial (Chengappa et al. 1986), así como en animales criados para laboratorio (DiGiacomo et al. 1983; Barnum, 1990; Frost $E$ Adler, 2000). En general, se sostiene que la $P$. multocida es el patógeno más frecuentemente aislado de las entidades arriba descritas en conejo. Sin embargo, igualmente se reportan aislamientos simultáneos de Bordetella bronchiseptica (Flatt E Dungworth, 1971a y b; Deeb et al. 1990; Moreno, 1995), especialmente en las afecciones respiratorias; no obstante, el papel que juega cada una de ellas durante los procesos infecciosos no ha sido claramente definido. La P. multocida y la $B$. bronchiseptica son consideradas parte de la flora normal de las vías aéreas superiores en conejos; sin embargo, factores ambientales macro, como el clima y el ambiente de los galpones y factores micro, como el ambiente de las mucosas de las vías aéreas, favorecerían su virulentación y patogenización en el hospedero (Bonilla E García-Delgado, 1993; Deeb \& DiGiacomo, 2000).

La prevalencía de la infección con $P$. multocida varía dependiendo de la edad, el sexo y el estado sanitario de los conejos. DiGiacomo et al. (1983) demostraron cómo en general la infección natural con P. multocida aumentó en un $4 \%$ en conejos de 12 semanas a un $23 \%$ en conejos de 22 semanas y hasta en un $72 \%$ en conejos adultos. Huertas \& Iregui (1996) reportaron que los animales a los 49 días de edad tenían un periodo crítico en el cual sufrían la enfermedad más frecuentemente; sin embargo, en este estudio no fue posible discriminar si la infección era debida a $P$. multocida, $B$. bronchiseptica o a ambas. En un estudio realizado en una granja de la Sabana de Bogotá, se realizaron aislamientos en conejos sanos de distintas edades comenzando desde neonatos hasta animales enfermos, se encontró que la $B$. bronchiseptica se aislaba prácticamente de cualquier animal sano después de los 21 días de edad y, en el $80 \%$ de los enfermos, mientras que la presencia de $P$. multocida, se limitaba a los conejos enfermos, aislándose en el $49 \%$ de los animales con signos de la enfermedad (Moreno, 1995).

Poco se conoce acerca de la patogénesis de las enfermedades inducidas por $P$. multocida. Una de las razones es la ausencia de un modelo experimental reproducible (Dowling et al. 2002). En general existen muy pocos estudios dirigidos a entender la epidemiología y las bases moleculares de la virulencia de las cepas asociadas con las enfermedades inducidas por estos patógenos en las diferentes especies animales (Davies et al. 2004).

\section{ENFERMEDAD RESPIRATORIA DE LOS CONEJOS}

Aunque no se ha determinado claramente cual es el conjunto de factores que predisponen el inicio de la enfermedad, se considera que la edad de los conejos es un punto clave en el desarrollo de ésta. Generalmente, los gazapos son infectados por la madre, posteriormente, la resistencia natural disminuye y la inmunidad de tipo pasivo va decayendo, observándose un aumento notable en la morbilidad después de las ocho semanas (Sánchez et al. 2000). En las hembras, las mucosas oral, conjuntival y vaginal parecen ser la principal vía de ingreso de la bacteria (Kpodekon et al. 1999; Deeb E DiGiacomo, 2000). Algunos autores reportan que éstas 
resultan ser más susceptibles, generalmente debido a la manipulación a que son sometidas como reproductoras (Sánchez et al. 2000).

Estudios serológicos e inmunohistoquímicos han demostrado un comportamiento variable de los anticuerpos en animales que sobreviven a la enfermedad. La respuesta de IgM se da desde el primer día de detección de signos hasta los días 14 - 21 y tiene caída precipitada hacia el día 28; para IgG, la respuesta es más tardía, iniciando el día siete aproximadamente, hasta el día 28 (Glass $\mathcal{E}$ Beasley, 1989; Takashima et al. 2001).

El complejo respiratorio de los conejos tiene varias formas de presentación. En los casos septicémicos predomina la fiebre, polipnea, disnea, cianosis de las mucosas y orejas y dilatación abdominal; algunos animales pueden morir sin síntomas aparentes (Glavits \& Magyar, 1990; Kpodekon et al. 1999). El cuadro respiratorio es la forma más común, tal vez por ser la más visible. Las vías aéreas superiores son las más frecuentemente involucradas (rinitis, sinusitis y traqueitis), se observa el pelo del hocico y de las extremidades anteriores húmedos como consecuencia de la descarga de un exudado mucopurulento y de los frecuentes estornudos, signos que pueden llegar a evolucionar hasta cuadros crónicos en los que finalmente se desarrolla destrucción de los cornetes nasales (Kpodekon et al. 1999). La conjuntivitis es una manifestación común de la enfermedad de las vías aéreas altas y obedece a infección por la contigüidad de los conductos nasolagrimales (Deeb E DiGiacomo, 2000). El curso hacia el tracto respiratorio bajo, se manifiesta clínicamente por fatiga, sonidos estertorosos en la traquea, dificultad respiratoria, cianosis de orejas y mucosas. A la auscultación, se puede detectar porciones del pulmón con sonidos ausentes muy probablemente por la presencia de abscesos (Deeb E DiGiacomo, 2000).

En la forma rinítica hay un pequeño exudado nasal hasta descarga nasal franca, mal estado general, depresión, anorexia y muerte (Al-Haddawi et al. 1999; Kpodekon et al. 1999; Deeb \& DiGiacomo, 2000). La forma pleuroneumoníca es la fase final de evolución de la forma respiratoria inferior y se caracteriza por hiperemia, disnea y por ser rápidamente mortal (Kpodekon et al. 1999).

Pasteurella multocida: En general, se sostiene que es el patógeno más frecuentemente asociado con entidades, como mastitis, otitis, neumonía, rinitis y sinusitis en conejo; sin embargo, se reportan aislamientos simultáneos de B. bronchiseptica, especialmente, en las afecciones respiratorias (Deeb et al. 1990; Moreno, 1995). No obstante, el papel que desempeña cada uno de estos microorganismos durante los procesos infecciosos respiratorios no ha sido claramente definido (Flatt $\mathcal{E}$ Dungworth, 1971a y b; DiGiacomo et al. 1983; Deeb et al. 1990; Kpodekon et al. 1999).

La P. multocida, una especie heterogénea encapsulada de bacterias Gram negativas es un organismo comensal de muchas especies animales (Harper et al. 2003). Bajo condiciones predisponentes, es el agente etiológico de un amplio rango de infecciones económicamente importantes en animales domésticos (Davies et al. 2004). El patógeno es un cocobacilo bipolar, no motil, oxidasa $(+)$, catalasa $(+)$, indol (+) ó (-), del cual se han descrito cinco tipos capsulares (A, B, D, E y F) y 16 serotipos somáticos (Carter, 1988). Posee factores que contribuyen a su virulencia, cuya expresión obedece, a su vez, a la expresión de genes involucrados con la biosíntesis, metabolismo y adquisición de nutrientes necesarios para la bacteria, los cuales proporcionarían información acerca de la interacción con el hospedero, durante la cual el microorganismo activaría señales de inducción génica que, finalmente, mediarían en los mecanismos de sobrevivencia e invasión a otros sitios anatómicos del hospedero (Hunt et al. 2001).

En el conejo solo las propiedades antigénicas o de serotipo no son suficientes para caracterizar las cepas de pasteurellas bien sea según su capacidad patogénica o según su origen epidemiológico; las cepas de un mismo serotipo pueden tener patogenicidad diferente. Rideaud $\varepsilon$ Coudert (1992) han demostrado que las cepas sin la enzima ornitin-decarboxilasa (ODC), cualquiera que sea el diámetro de la colonia, son poco o nada patógenas; este criterio es más fiable que la medida de la colonia para prever la patogenicidad de las cepas independientemente de las condiciones de cultivo (Rideaud \& Coudert, 1992; Kpodekon et al. 1999).

Factores de virulencia: Se describen varios factores de virulencia aún no bien caracterizados, entre los cuales están el polisacarido capsular, el lipopolisacarido, la proteína externa de membrana ligadora de hierro, la proteasa, la neuraminidasa y la porina (Rideaud $\&$ Coudert, 1992; Boyce $E$ Adler, 2000). Chen et al. (2003) han 
propuesto, más recientemente, el sistema de metilación de la adenina, el cual regula la expresión de un gen de virulencia denominado Dam en esta bacteria, al igual de lo demostrado en otras Gram negativas.

La estructura capsular determina el serotipo de la bacteria. Los polisacaridos son el mayor componente de ella y, en algunos casos, están acompañados de lipoproteínas. En el serotipo A, la cápsula esta compuesta en su mayoría de ácido hialuronico sensible a la acción de la enzima hialuronidasa, lípidos y proteínas. La cápsula no exhibe por sí sola propiedades antifagociticas, ya que no tiene propiedades antigénicas, pero el extracto capsular bacteriano contiene un factor proteico $(300 \mathrm{kD})$ capaz de inhibir los fagocitos bovinos (Seleim, 1996, Watt et al. 2003). Las cápsulas de las cepas aviares proveen protección contra la acción del complemento pero esto no determina la relación del organismo con las células fagocíticas, encontrándose igualmente reacción inflamatoria. Existen diferentes resultados en cuanto a la mayor o menor adhesividad o mayor susceptibilidad a la fagocitosis al ser retirada la cápsula (Champlin et al. 2002; De-Angelis et al. 2002).

En conejos, los aislamientos más frecuentes de $P$. multocida en cuadros respiratorios son los serotipos A y D (DiGiacomo et al. 1989, Borkowska-Opacka et al. 1995), de los antígenos somáticos 3 y 12 (Flatt $E$ Dungworth, 1971a; Brodgen et al. 1989; Manning, 1982; BorkowskaOpacka et al. 1995). Se han descrito características más específicas para algunas de las variedades anteriores, por ejemplo, las cepas del grupo A:3 son capsuladas, poseen fimbria, no hemaglutinan el grupo sanguíneo $O$ humano y no producen toxina dermonecrótica; mientras las del serotipo D: 1 son acapsulares, poseen fimbria, hemaglutinan el grupo sanguineo $O$ humano y producen toxina dermonecrótica (Al-Haddawi et al. 2000).

Las cepas del serotipo A son más adhesivas a la superficie mucosa del hospedero que las del serotipo D aunque ambas poseen fimbrias que expresan en su membrana (Glorioso et al. 1982; Deeb E DiGiacomo, 2000). La presencia de fimbrias fue demostrada en $P$. multocida aislada de células faringeas de conejo, células tonsilares y nasales de cerdo, y nasales y traqueales de terneros. La adhesión de $P$. multocida del serotipo A es inhibida por la $\mathrm{N}$-acetil D-glucosamina; se sugiere que este amino-azúcar sería el receptor de las células del hospedero, al cual se une la fimbria de la bacteria. Algunos resultados han confirmado el papel de la fimbria y/o el material capsular en los procesos de adherencia utilizando anticuerpos contra ambas estructuras, aquellos inhibieron específicamente la capacidad de adherencia del microorganismo (Seleim, 1996; Esslinger et al. 1994). Investigaciones en porcinos muestran que algunas cepas del serotipo A son más adhesivas y no tienen necesariamente la capacidad de colonizar la mucosa nasal normal, por lo tanto, describen una pobre correlación entre la presencia de fimbria y la producción experimental de rinitis atrófica en cerdos (Esslinger et al. 1994).

En P. multocida, tipo D, las hemaglutininas son cruciales en colonización de la nasofaringe y traquea en cerdos, jugando la fimbria y la toxina dermonecrótica un papel significativo (Boyce $\&$ Adler, 2000). La toxina termolabil (PTM), igualmente involucrada con procesos respiratorios severos, es una proteína citoplasmática con un peso molecular entre 149 y $160 \mathrm{KD}$, se le ha demostrado principalmente en cepas del tipo capsular D y algunas toxigénicas del tipo A (Suckow et al. 1996).

Las proteínas de membrana externa (OMP) de las bacterias Gram negativas son la interfase entre el patógeno y el hospedero y juegan un papel esencial en la interacción inicial de estos en los procesos de enfermedad. Dentro de las anteriores, se menciona la proteína ligadora de hemoglobina, por intermedio de la cual la bacteria captura el hierro en ambientes escasos del mismo en forma libre. Este es uno de los elementos esenciales en las rutas metabólicas de transporte de electrones del patógeno. Mientras que algunos microorganismos liberan sideroforos para quelar el hierro libre, la $P$. multocida, principalmente las cepas patogénicas, expresan en su superficie la proteína, permitiendo su interacción con el sustrato. La proteína ligadora de hemoglobina está conformada por dos subunidades TbpA y TbpB para la transferrina y Lbpa y LbpB para lactoferrina; son codificadas por un grupo de tres genes (box ton B) que al sufrir ligeras variaciones en la secuencia, aumentarían la variabilidad y, por ende, la evasión a la respuesta inmune (Confer et al. 2001; Bosch et al. 2002).

Los aislamientos de $P$. multocida varían en su habilidad para producir enfermedad en animales. Algunas están asociadas principalmente con enfermedades del tracto respiratorio alto mientras que otras pueden causar septicemia, abscesos y neumonía. Con el objeto de producir infección la bacteria puede colonizar la mucosa 
respiratoria y los organismos que pueden realizar esta colonización frecuentemente producen sialidasas. Estas enzimas han mostrado una actividad glicolítica sobre las mucinas, la cual libera los residuos de ácido síalico terminales para ser usados como fuente de carbono por la bacteria. La sialidasa es una enzima glicolítica únicamente extracelular producida por la $P$. multocida, sugiriendo que esta enzima probablemente juega un papel central en la habilidad de la $P$. multocida para colonizar las mucosas. Muchos aislamientos poseen dos genes de silaidasas que codifican enzimas con diferentes especificidades de substrato, por ejemplo, algunas cepas mutantes deficientes en sialidasas tienen una habilidad reducida para replicarse usando los glicoconjugados, como fuentes de carbono. La expresión de la sialidasa ha mostrado ocurrir durante la infección, más aun es probable que el huésped haya sido expuesto a la proteína durante la colonización de la bacteria (Sánchez et al. 2004).

Lipolisacarido: El lipopolisacárido (LPS) es el componente más abundante en la cápsula de las bacterias Gram negativas, considerado como uno de los más importantes productos bacterianos dotado con propiedades inmunomodulatorias y proinflamatorias, reconocido ampliamente como determinante de virulencia y es, tal vez, el mediador más importante del shock endotóxico (De Boer et al. 1992). El LPS es un mediador entre la bacteria y el hospedero y favorece la colonización e infección a través de mecanismos, como mimetismo molecular, variaciones antigénicas y bloqueo de anticuerpos (Harvill et al. 2000).

Durante la infección por bacterias Gram negativas, el LPS estimula el sistema inmune innato, haciendo que la respuesta inflamatoria juegue un papel crítico en la ayuda de la limpieza de la bacteria y prevención de la infección. Esta respuesta inicial contra las bacterias Gram negativas puede ser iniciada por un amplio número de componentes bacterianos, siendo más potente el lípido A. Si la respuesta inicial del hospedero es incapaz de limpiar la bacteria y la infección toma su curso, las grandes cantidades sistémicas de LPS pueden resultar en shock endotóxico, en el cual, una sobreproducción de mediadores inflamatorios, causan daño a los tejidos, shock séptico, falla orgánica y muerte (Raetz E Whitfield, 2002).

Específicamente, se considera que el LPS de la $P$. multocida juega un rol importante en la patogénesis de la enfermedad, como uno de los más importantes factores de virulencia (Michael et al. 2005). Recientemente, se ha mostrado que el LPS de $P$. multocida está involucrado en la adhesión de neutrófilos al endotelio y la transmigración de éstos a través de las células endoteliales (Galdiero et al. 2000). El estudio de varias cepas de $P$. multocida aisladas de distintas especies animales confirmó que el LPS de esta bacteria es ligeramente similar al de las Enterobacteriaceas (Horadagoda et al. 2001). Cepas de pasteurellas provenientes de cerdos con rinitis atrófica (serotipo capsular D) mostraron, por lo menos, seis diferentes tipos electroforéticos de LPS y estos frecuentemente coincidían con la presencia de ciertas OMP y la presencia o ausencia de carácter patogénico de la cepa, por lo tanto, se considera que en las OMP y el LPS son un factor de virulencia que se encuentra ligado y responde a la activación de un mismo grupo de genes. Sin embargo, existen reportes contradictorios acerca de la capacidad endotóxica del LPS de P. multocida. Por ejemplo, el LPS aislado del serotipo B2 muestra ser endotóxico y al ser administrado intravenosamente es capaz de reproducir los signos clínicos de la septicemia hemorrágica en búfalo (Horadagoda et al. 2001). Una modificación en la estructura de LPS claramente afecta la viabilidad de la $P$. multocida in vivo. La modificación genética del ensamblaje del LPS de $P$. multocida comprobó que cepas con LPS truncados son menos virulentas e incapaces de sobrevivir en el hospedero natural (Harper et al. 2003).

Existen diferencias de susceptibilidad entre especies animales aun con el mismo LPS, así los pavos son relativamente resistentes a los efectos letales del LPS del serogrupo A, aunque la reacción inflamatoria y las lesiones hepáticas microscópicas son similares a las de los hospederos mamíferos. En contraste, los embriones de pollo y de ratones han demostrado ser altamente susceptibles a los efectos tóxicos del LPS de P. mutocida (Ganfield et al. 1976). La respuesta en ratones y en conejos inoculados con LPS de P. multocida purificado es muy pobre en cuanto a la formación de anticuerpos y, por lo tanto, no ofrece protección contra la pasteurelosis. El mismo LPS inyectado en pollos induce buena respuesta de anticuerpos y produjo protección pasiva contra la enfermedad (Ramdani E Adler, 1991).

Anticuerpos reactivos a LPS de cepas del serotipo A3 han mostrado protección parcial contra la infección en conejos y ratones. El LPS parece igualmente jugar un 
papel implícito en la protección contra el serotipo B. Se demostró que el LPS estimula la liberación de TNF- $\alpha$, inteleukinas y muchos otros factores de los macrófagos alveolares, los cuales mostraron tener acción mitogénica. La acción mitogénica fue explicada a través del daño en los mecanismos de las células del hospedero, liderados por la proliferación celular y el bloqueo de la apoptosis (Bienhoff et al. 1992; Horadagoda et al. 2001; Lax \& Thomas 2002).

Efecto de la $P$. multocida y su LPS sobre epitelio respiratorio: El complejo Pasteurella-Bordetella es un modelo representativo de enfermedad respiratoria, en la cual, los microorganismos y/o sus toxinas (lipopolisacárido) se pueden diseminar en el hospedero. La P. multocida es un organismo comensal de muchas especies animales (Harper et al. 2003) y, bajo condiciones predisponentes, es el agente etiológico de un amplio rango de infecciones (Davies et al. 2004).

Varios autores han descrito cambios microscópicos en el tracto respiratorio alto de conejos con la forma natural de la enfermedad encontrando hiperactividad de las células caliciformes, necrosis, vacuolización intracitoplasmática e infiltración de PMN (Botero E Iregui, 1999; Al Haddawi et al. 1999; Esquinas et al. 2004). Esquinas (2007) y Gallego (2007) han evaluado cualitativa y cuantitativamente las lesiones en experimentos in vitro realizados exponiendo, individualmente, septos de feto de conejo a P. multocida, B. bronchiseptica y sus lipopolisacáridos, confirmado que tanto las bacterias individualmente como sus LPS pueden ocasionar cambios tempranos en este tipo de tejidos.

En trabajos con P. multocida y su LPS (Esquinas, 2007), se hicieron evidentes cambios de dos tipos: degenerativos y de hiperactividad. Entre los primeros, se encuentran lesiones, como aumento de los espacios intercelulares, vacuolización citoplasmática, descamación celular y células en necrosis o apoptosis. Estos hallazgos coinciden, con lo encontrado por otros autores, tanto in vitro como in vivo. Por ejemplo, Al Haddawi et al. (1999) describen en conejos infectados, experimentalmente con $P$. multocida, cambios degenerativos en las células ciliadas, como vacuolización citoplasmática, pérdida de cilias y necrosis tanto en cavidad nasal como en traquea de animales infectados experimentalmente, así como hipertrofia de las células caliciformes. Esquinas et al. (2004) encontraron cambios similares en animales con la forma natural del complejo Pasterurella-Bordetella en conejos, en los cuales se demostró el mismo tipo de cambios celulares degenerativos e hiperactividad de células caliciformes asociadas siempre con infiltración de PMN en el epitelio y en la lámina propia.

Varios autores atribuyen muchos de estos cambios a la acción directa de la bacteria con algunos de sus factores de virulencia, como las porinas. En el caso de la $P$. multocida, se describe la proteína $\mathrm{H}(\mathrm{OmpH})$, la cual actúa como formadora de canales transmemebranales, los cuales pueden causar un daño homeostático en las células induciendo su hinchamiento, degeneración y muerte (Luo et al. 1997, Gorga et al. 2001).

Igualmente, se ha demostrado que el LPS es capaz de inducir tanto necrosis como apoptosis en varios tipos de tejidos y células por vías diferentes. El proceso de apoptosis puede ser tanto bioquímico como morfológicamente distinto al de la necrosis y contribuye al daño de los tejidos. Las vías de señalización que promueven la apoptosis, se pueden dividir en dos: la vía intrínseca (mediada por receptores de membrana) y la vía extrínseca (dependiente de la mitocondria). Los receptores de membrana pertenecen a una superfamilia de los del TNF y terminan en la activación de la vía de las caspasas. La vía extrínseca es dependiente de la permeabilidad de la membrana mitocondrial y no requiere de receptores para ser activada (Neff et al. 2006). Galdiero et al. (2000), demostraron que el LPS y las porinas derivadas de la $P$. multocida, inducían apoptosis en células espermáticas por daño directo sobre su membrana externa. En nuestro caso la apoptosis y la necrosis, se pueden deber tanto a la acción directa de la bacteria como la del LPS y, de acuerdo con algunos autores, este tipo de cambios tempranos ocurren en los epitelios, como mecanismo de defensa para eliminar el agente injuriante (Williams et al. 1994, Neff et al. 2006), sin embargo, si el agente persiste el daño, se puede extender y hacerse irreversible.

El segundo tipo de cambios es de hiperactividad, los cuales se evidenciaron en las células caliciformes (CC), que respondieron tanto a la $P$. multocida como a su LPS. Se encontró un aumento en la actividad de estas células, la cual se reflejó en un aumento del tamaño, en el contenido intracitoplasmático de moco, presencia de células expulsando su contenido y por un incremento en el acúmulo de moco secretado en la luz del órgano, al cual se asociaban tanto bacterias como células 
descamadas y LPS. De la misma manera, trabajos previos en fosa nasal de conejos con la forma natural de la enfermedad respiratoria por $P$. multocida y/o $B$. bronchiseptica documentaron alteraciones similares de las CC (Doncel, 2004). Estos autores propusieron que si bien la participación de las CC en estos procesos infecciosos puede deberse al aumento del número de dichas células, tal vez su mayor significado estaría dado por una mayor actividad sintética y secretora de las mismas. Se sugirió que el aumento tanto en el número como en la función de estas células en la fosa nasal de animales expuestos a las bacterias y/o sus LPS, representaría un reto para la sobrevivencia de los conejos, toda vez que una exagerada producción de moco en este sitio de escaso diámetro, poco explandible y de necesaria rápida circulación del aire, posiblemente llevaría al taponamiento. Se ha demostrado en varios trabajos que la instilación intratraqueal o intranasal de LPS lleva al incremento de la cantidad de moco secretado (Beckmann et al. 2002; Wagner et al. 2003).

Este cambio ha sido reportado por otros autores en animales expuestos a LPS (Harkema \& Hotchkiss, 1991; Shimizu et al. 1996). La hipertrofia, hiperplasia y/o metaplasia de las células secretoras en el epitelio y las glándulas submucosas se ha asociado con la hipersecreción de moco y es uno de los principales cambios patológicos encontrados en inflamaciones de las vías aéreas, como rinitis, sinusitis y traqueobronquitis. Harkema $\mathcal{E}$ Wagner (2002) demostraron que la instilación intranasal de endotoxina induce una inflamación caracterizada por el incremento en la cantidad de mucosustancias e infiltración de PMN (polimorfos nucleares).

El mecanismo por el cual la endotoxina promueve la producción de moco no es bien entendido. Experimentalmente, se demostró que los cambios en el epitelio disminuyeron al bloquear el ingreso de neutrófilos y que al instilar elastasa de PMN intranasalmente se producían lesiones muy similares, demostrando que células inflamatorias, como los neutrófilos, podrían estar estimulando las modificaciones epiteliales inducidas por la endotoxina (Harkema E Hotchkiss, 1991; Shimizu et al. 1996; Shimizu et al. 2003). Agusti et al. (1998) demostraron un importante papel de los neutrófilos en la degranulación de las células caliciformes, ésta se explica por la presencia de elastasa en las regiones donde se encontraron las células caliciformes degranulándose o vertiendo su contenido al lumen; la elastasa se encuentra contenida en los gránulos azurófilos de los neutrófilos, $\mathrm{y}$ ha demostrado ser un potente secretagogo sobre las glándulas de las vías aéreas y sobre las células caliciformes.

En varios trabajos in vivo se demuestra cómo la endotoxina promueve la producción de mediadores, como TNF- $\alpha$, IL-1, factor activador plaquetario y elastasa derivada de neutrófilos que inducen de la expresión de genes que codifican para la producción de mucina o inducen la metaplasia celular mucosa. Por el contrario, en otros experimentos con cultivos celulares o explantes nasales, la endotoxina indujo la expresión de genes de mucina sin que fueran necesarios otros mediadores secundarios (Beckmann et al. 2002; Wagner et al. 2003).

Estos hallazgos pueden en parte, explicarse por la acción del LPS de $P$. multocida A:3, el cual mostró ser tóxico para embriones de ratones y de pollo, causando una mortalidad del $50 \%$ con una dosis de $250 \mathrm{mg}$ (Ganfield et al. 1976). Adicionalmente es reportado por varios autores la respuesta del tracto respiratorio al LPS como una repuesta aumentada de células y de glándulas mucosas y una inflamación aguda compuesta principalmente por PMN (Lefort et al. 1998; Harkema \& Wagner, 2002; Brass et al. 2004). En el caso de P. multocida no se han encontrado reportes donde se describan cambios en la mucosa respiratoria inducidos por su LPS, exclusivamente.

En estudios realizados con P. multocida, se ha demostrado que la principal causa inflamatoria son los PMN, además que la han relacionado con varias de las lesiones y cambios encontrados en los animales inoculados (Al Haddawi et al. 1999; Esquinas et al. 2004). Venaille et al. (1995) postulan que los PMN serían capaces de inducir descamación de las células epiteliales una vez son estimulados con agentes activadores de PMN como el LPS bacteriano. Tal descamación sería mediada a través de enzimas denaturalizantes e hidrolizantes de los PMN las que ejercerían acción sobre proteínas, como la lámina, la fibronectina y el colágeno tipo IV.

Distintos autores han demostrado que las principales células inflamatorias en ratas expuestas intratraqueal o intranasalmente a distintas dosis de LPS de Pseudomonas aeruginosa (20 a 1000mg) son neutrófilos y macrófagos, mientras que los linfocitos y los esosinofilos 
no estuvieron presentes (Foster et al. 2003; Wagner et al. 2003). Según ellos, las dosis entre 20 a 100mg de endotoxina son suficientes para iniciar procesos de inflamación neutrofílica en el pulmón. Reustershan et al. (2006) mostraron por técnicas inmunohistoquimicas, como la migración tranendotelial y transepitelial de PMN en respuesta al LPS, puede ser mediada por la proteína de membrana CXCR2, la cual se expresa principalmente en los capilares, sitio donde residen los PMN.

En conclusión, debido a la amplia distribución de estos agentes infecciosos en varias especies animales, así como la ausencia de modelos de estudio de la fisiopatología de esta clase de enfermedades es muy importante contribuir en el conocimiento de sus comportamientos y su interacción con el hospedero para desarrollar mecanismos de prevención, control y tratamiento, aplicables en varias patologías.

\section{BIBLIOGRAFÍA}

AL-HADDAWI, M.; JASNI, S.; ZAMRI-SAAD, M.; MUTALIB, A.; SHEIKH, O. 1999. Ultrastructural pathology of the upper respiratory tract of rabbits experimentally infected with Pasteurella multocida A:3. Res. Vet. Sci. (Inglaterra). 67(2):163-170.

AL-HADDAWI, M.; JASNI, S.; ZAMRI-SAAD, M.; MUTALIB, A.; ZULKIFLIZ, I.; SON, R. 2000. In vitro study of Pasteurella multocida adhesion to trachea, lung and aorta of rabbits. The Vet. J. (Inglaterra). 159(3):274-281.

AGUSTI, C.; TAKEYAMA, K.; CARDELL, L.; UEKI, I.; LAUSEIER, J.; LOU, Y.; NADEL, J. 1998. Goblet cell degranulation after antigen challenge in sensitized guinea pigs role of neutrophils. Am. J. Respir. Crit. Care Med. 158 (4):1253-1258.

ANDREWS, G.; KENNEDY G. 1997. Respiratory diagnostic pathology. Vet. Clin. North America. Food Anim. Pract. 13:515-547.

BARNUM D. 1990. Socioeconomic significance of the HAP group. Review. Can. J. Vet. Res. 54 Suppl: S1-5.

BECKMANN, N.; TIGANI, B.; SUGAR, R.; JACKSON, A.; JONES, G.; MAZZONI, L.; FOZARD, R. 2002.
Noninvasive detection of endotoxin-induced mucus hypersecretion in rat lung by MRI. Am. J. Physiol. Lung Cell. and Molecular Physiol. 283(1): L22-L30.

BIENHOFF, S. E.; ALLEN, G.K.; BERG, J.N. 1992. Release of tumor necrosis factor-alpha from bovine alveolar macrophages stimulated with ovine respiratory viruses and bacterial endotodxins. Vet. Immunol. Immunopathol. (EEUU). 30:341-357.

BONILLA, F.; GARCÍA-DELGADO, G. 1993. Adherence of Pasteurella multocida to rabbit respiratory epithelial cells in vitro. Rev. Latinoam. Microbiol. (México). 35(4):361-369.

BOTERO, L.; IREGUI, C. 1999. Caracterización de la interrelación entre la Pasteurella multocida y la Bordetella bronchiseptica con células epiteliales de la cavidad nasal y la nasofaringe durante el curso de la neumonía enzoótica de los conejos. Rev. Med. Vet. Zoot. (Colombia). 46:21-39.

BORKOWSKA-OPACA, B.; RUTKOWSKA-JURGA, I.; TRUSZYNKY, M. 1995. Determination of the serotypes of Pasteurella multocida strains isolated from rabbit. Bull. Vet. Ins. Pulawy. (Bulgaria) 39:9-12.

BOSCH, M.; GARRIDO, E.; LLAGOSTERA, M.; PÈREZ DE ROZAS, A.; BADIOLA, I.; BARBÉ, I. 2002. Characterization of the Pasteurella multocida hgbA gene encoding a hemoglobin-binding protein. Infect. Immun. (EEUU). 70(11):5955-5964.

BOYCE, J.; ADLER, B. 2000. The capsule is a virulence determinant in the pathogenesis of Pasteurella multocida M1404 (B:2). Infect. Immun. 68:34633468.

BRASS, D.; SAVOV, J.; WHITEHEAD, G. 2004. LPS binding protein is important in the airway response to inhaled endotoxin. J Allergy Clin Immunol. (EEUU). 114(3):586-92.

BROGDEN, K.A.; ADLAM C., R.C.; KNIGHTS, J.M.; ENGEN. R.L. 1989. Effect of P. haemolytica (Al) capsular polysaccharides on sheep lung in vivo and on pulmonary surfactant in vitro. Am. J. Vet. Res. 50:555-559. 
BROCKMEIER, S.; REGISTER, K. 2000. Effect of temperature modulation and bvg mutation of Bordetella bronchiseptica on adhesion, intracellular survival and cytotoxicity for swine alveolar macrophages. Vet. Microbiol. (Holanda). 73(1):1-12.

BUREAU, F.; DETILLEUX, J.; DORTS, T.; UYSTEPRUYST, C.; COGHE, J.; LEROY, P.; LEKEUX, P. 2001. Spirometric performance in belgian blue calves: I. Effects on economic losses due to the bovine respiratory complex. J. Anim. Sci. (EEUU). 79(5):1301-1304.

CARTER, G. 1988. Serological classification of pasteurella. Vet. Rec. (Inglaterra). 26;122(13):311.

CHAMPLIN, F.R.; SHRYOCK T.R.; PATTERSON, C.E.; AUSTIN, F.W.; RYALS, P.E. 2002. Prevalence of a novel capsule-associated lipoprotein among pasteurellaceae pathogenic in animals. Curr. Microbiol. (Francia) 44(4):297-301.

CHENGAPPA, M; CARTER, B; BAILIE W. 1986. Identification of type D Pasteurella multocida by counterimmunoelectrophoresis. J Clin Microbiol. (EEUU). 24(5):721-723.

CHEN, L.; PAULSEN, D.; SCRUGGS, D.; BANES, M, REEKS B.; LAWRENCE M. 2003. Alteration of DNA adenine methylase (Dam) activity Pasteurella multocida causes increased spontaneous frequency and attenuation in mice. Microbiol. (EEUU). 149: 2283-2290.

CONFER, A. W.; SUCKOW, M. A.; MONTELARGO, M.; DABO, S. M.; MILOCIO, L. J.; GILLESTIE, A.; MEREDIYH, G. L. 2001. Intranasal vaccination of rabbits with Pasteurella multocida outer membranes that express iron-regulated proteins. Am J. Vet. Res. 62(5):697-703.

COY, S.; LEE, M. D.; SANDER, J. 1997. Intrastrain variation of lipopolysaccharide of Pasteurella multocida in turkeys. Am J Vet Res. 58(7):755-759.

DAVIES, R.; MACCORQUODALE, R.; REILLY, S. 2004. Characterisation of bovine strains of Pasteurella multocidaand comparison with isolates of avian, ovine and porcine origin. Vet. Microb. (Holanda). 99:145-158.

DEBOER, J.; WOLBINK, G.; THIJS, L.; BAARS, J.; WAGSTAFF, J.; HACK, E. 1992. Interplay of complement and cytokines in the pathogenesis of septic shock. Immunopharmacol. (EEUU) 24:135-148.

DEEB, B.; DIGIACOMO, R.; BERNARD, B.L.; SILVERNAGEL, S. 1990. Pasteurella multocida and infections in rabbits. J. Clin. Microbiol (EEUU). 28:70-75.

DEEB, B.; DIGIACOMO, R. 2000. Respiratory diseases of rabbits. The veterinary clinics of North America. Exotic An. Pract. 3(2):465-480.

DE-ANGELIS, P.; GUNAY, N.; TOIDA, T.; MAO, W.; LINHARDT, R. 2002. Identification of capsular polysaccharides of type $\mathrm{D}$ and $\mathrm{F}$ Pasteurella multocida as unmodified heparin and chondroitin, respectively. Carbohydr. Res. (EEUU). 337(17):1547-1548.

DIGIACOMO, R.; GARLINGHOUSE, L.; VAN HOOSIER, G. 1983. Natural history of infection with Pasteurella multocida in rabbits. J. Am. Vet. Med. Assoc. 183(11):1172-1175.

DIGIACOMO, R.; DEEB, B.J.; GIDDENS, W.E.; BERNARD, B.L.; CHENGAPPA, M.M. 1989. Atrophic rhinitis in New Zealand white rabbits infected with Pasteurella multocida. Am. J. Vet. Res. 50(9):1460-1465.

DONCEL, B. 2004. Cuantificación y caracterización histoquímica preliminar de las células caliciformes en fosa nasal y nasofaringe de conejos sanos y con el síndrome de neumonía enzoótica. Tesis de Maestría. Facultad de Medicina Veterinaria y de Zootecnia. U. Nacional de Colombia.

DOWLING, A.; HODGSON, J.; SCHOCK, A.; ECKERSALL, P.; MCKENDRICK, I. 2002. Experimental induction of pneumonic pasteurellosis in calves by intratracheal infection with Pasteurella multocida biotype A:3. Res. Vet. Sci. (Inglaterra). 73(1):37-44.

ESQUINAS, P.; IREGUI, C.; BOTERO, L. 2004. Comparación ultraestructural e inmunohistoquimica del 
epitelio nasal de conejos sanos y con el complejo respiratorio Pasteurella-Bordetella. Memorias IV Reunión Anual de Patología Veterinaria. La Plata. Argentina. IV RAPAVE: p.56.

ESQUINAS, P. 2007. Interacción in vitro entre la Pasteurella multocida y/o su lipopolisacárido con epitelio de septo nasal de conejo. Tesis de Maestría. Facultad de Medicina Veterinaria y de Zootecnia. U. Nacional de Colombia.

ESSLINGER, J., SELEIM, R.S.; HERRMANN, G.; BLOBEL, J. 1994. Adhesin of Pasteurella multocida to Hela cells and to macrophages of different animal species. Rev. Med. Vet. (Inglaterra) 145(1): $49: 53$.

FLATT R. ; DUNGWORTH D. 1971a. Enzootic pneumonia in rabbits: naturally occurring lesions in lungs of apparently healthy young rabbits. Am J Vet Res, 32(4): 621-626.

FLATT R. ; DUNGWORTH D. 1971b. Enzootic pneumonia in rabbits: microbiology and comparison with lesions experimentally produced by Pasteurella multocida and a chlamydial organism. Am J Vet Res. 32(4):627-637.

FOSTER, J.E., GOTT, K.; SCHUYLER, M.R.; KOZAK, W.; TESFAIGZI, Y. 2003. LPS-induced neutrophilic inflammation and $\mathrm{Bcl}-2$ expression in metaplastic mucous cells. Am. J. Physiol. Lung Cell. Molecular Physiol. 285(2):L405-L414.

FROST, A.; ADLER, B. 2000. Pasteurella multocida: the elusive determinants of virulence and immunity. Vet. Microb. 72:1-2.

GALDIERO, M.; FOLGORE, A.; UNZZO, I.; GALDIERO, E. 2000. Neutrophil adhesion and transmigration through bovine endothelial cells in vitro by protein $\mathrm{H}$ and LPS of Pasteurella multocida. Immunobiology (Alemania). 226:57.

GALLEGO, C. 2007. Interacción in vitro entre la Bordetella bronchiseptica y/o su lipopolisacárido con epitelio de septo nasal de conejo. Tesis de Maestría. Facultad de Medicina Veterinaria y de Zootecnia. U. Nacional de Colombia.
GANFIELD, D.; REBERS, P.; HEDDLESTON, K. 1976. Immunogenic and toxic properties of a purified lipopolysaccharide-protein complex from Pasteurella multocida. Infect. Immun. 14(4):990-999

GLASS, L.; BEASLEY, J. 1989. Infection with and antibody response to Pasteurella multocida and Bordetella bronchiseptica in immature rabbits. Lab. Anim. Sci. (EEUU). 39(5):406-410.

GLAVITS, R.; MAGYAR, T. 1990. The pathology of experimental respiratory infection with Pasteurella multocida and Bordetella bronchiseptica in rabbits. Acta. Vet. Hungarica. 38(3):211-215.

GLORIOSO, J.; JONES, G.; RUSH, H.; PENTLER, L.; DARIF, C.; COWARD J. 1982. Adhesion of type A Pasteurella multocida to rabbit pharyngeal cells and its possible role in rabbit respiratory tract infections. Infec. Immun. 35:1103-1109.

GRIFFIN, D. 1997. Economic impact associated with respiratory disease in beef cattle. Vet. Clin. North Am. Food Anim. Practice. 13(3):367-377.

GORGA, F.; GALDIERO, M.; BUOMMINO, E.; GALDIERO, E. 2001. Porins and lipopolysaccharide induce apoptosis in human spermatozoa. Clin. Diagn. Lab. Immun. (EEUU). 8(1):206-208.

HARKEMA, J.; HOTCHKISS, J. 1991. In vivo effects of endotoxin on nasal epithelial mucosubstances: quantitative histochemistry. Exp Lung Res. (EEUU). 17(4):743-61.

HARKEMA, J.; WAGNER, J. 2002. Non-allergic models of mucous cell metaplasia and mucus hypersecretion in rat nasal and pulmonary airways. Novartis Found Symp. (Inglaterra). 248:181-97; Discussion 197-200, 277-82.

HARPER, M.; BOYCE, J.; WILKIE, W.; ADLER, B. 2003. Signature-tagged mutagenesis of Pasteurella multocida identifies mutants displaying differential virulence characteristics in mice and chickens. Infect. Immun. 71(9):5440-5446.

HARVILL, E.; PRESTON, A.; COTTER, P.; ALLEN, A.; MASKELL, D.; MILLER, J. 2000. Multiple roles 
for Bordetella lipopolysaccharide molecules during respiratory tract infection. Infect. Immun. 68(12):6720- 6728.

HORADAGODA, N.U.; HODGSON, J.C.; MOON, G.M.; WIJEGARDANA, T.; ECKERSALL, P. 2001. Role of endotoxins in the pathogenesis of haemorrhagic septicaemia in buffalo. Microb. Pathol. (Inglaterra). 30(3):171-178.

HUERTAS, C.; IREGUI, C. 1996. Estudio prospectivo de la neumonía enzoótica por Pasteurella multocida y Bordetella bronchiseptica en dos granjas cunículas de la altillanura de Bogotá. Abstracts XV Congreso Panamericano de Ciencias Veterinarias. Campo Grande, Brasil.

HUNT, M.L.; BOUCHER, D.J.; BOYCE, J.D.; ADLER, B. 2001. In vivo-expressed genes of Pasteurella multocida. Infect. Immun. 69:3004-3012.

KPODEKON, M.; RIDEAUD, P.; COUDERT P. 1999. Pasteurellosis du lapin: revue. Rev. Méd. Vét. (Francia). 150:221-232.

LAX, A.J.; THOMAS, W. 2002. How bacteria could cause cancer: one step at a time. Trends Microbiol. (EEUU). 10(6):293-299.

LEFORT, J.; SINGER, M.; LEDUC, D.; RENESTO, P.; NAHORI, M.; HUERRE, M.; CREMINON, C.; CHIGNARD, M.; VARGAFTIG, B. 1998. Systemic administration of endotoxin induces bronchopulmonary hyperreactivity dissociated from TNF-alpha formation and neutrophil sequestration into the murine lungs. J Immunol. (EEUU). 1;161(1):474-80.

LUO, Y., GLISSON, J.R.; JACKWOOD, M.W.; HANCOCK, R.E.; BAINS, M.; CHENG, I.H.; WANG, C. 1997. Cloning and characterization of the major outer membrane protein gene $(\mathrm{ompH})$ of Pasteurella multocida X-73. J. Bacteriol. (EEUU). 179:78567864.

MANNING, P. 1982. Serology of Pasteurella multocida in laboratory rabbits: a review. Lab. Anim. Sci. 32:666-671.
MEYER, F.P. 1991. Aquaculture disease and health management. J. Anim. Sci. 69:4201-4208.

MICHAEL, F.; VINOGRADOV, E.; LI, J.; COX, A. 2005. Structural analysis of the lipopolysaccharide from Pasteurella multocida genome strain Pm70 and identification of the putative lipopolysaccharide glycosyltransferases. Glycobiology. (Inglaterra). 15(4):323-33.

MORENO, A. 1995. Aislamiento y tipificación de Pasteurella multocida y Bordetella bronchiseptica involucradas en el complejo neumónico de conejos en una granja de la Sabana de Bogotá. Tesis de Pregrado. Facultad de Medicina Veterinaria y de Zootecnia. U. N. de Colombia.

MURTAUGH, M.P.; BAARSCH, M.J.; ZHOV, Y.; SCAMURRA, R.W.; LIN, G. 1996. Inflammatory cytokines in animal health and disease. Vet. Immunol. Immunopathol. 54:45-55.

NEFF, S.; ZAGRAGGEN, B.; NEFF, T.; JAMNICKI-ABEGG, M.; SUTER, D.; SCHIMMER, R.; BOOY, C.; JOCH, H.; PASCH, T.; WARD, P.; BECK-SCHIMMER, B. 2006. Inflammatory response of tracheobronchial epithelial cells endotoxin. Am. J. Physiol. Lung Cell. Molecular Physiol. 290:L86-L96.

RAETZ, C.; WHITFIELD, C. 2002. Lipopolysaccharide endotoxins. Ann. Rev. Biochem. (EEUU). 71:635700 .

RAMDANI, B.; ADLER, B. 1991. Opsonic monoclonal antibodies against lipopolysaccharide (LPS) antigens of Pasteurella multocida and the role of LPS in immunity. Vet. Microbiol. (Holanda).15;26(4):335-47.

REUTERSHAN, J.; MORRIS, M.; BURCIN, T.; SMITH, D.; CHANG, D.; SAPRITO, M.; LEY, K. 2006. Critical role of endothelial CXCR2 in LPS induced neutrophil migration into the lung. J. Clin. Invest. (EEUU) 116:695-702.

RIDEAUD, P.; COUDERT, P.; MERCIER, P.; HERVOUET, P. 1992. A comparative study of the virulence of Pasteurella multocida from rabbits (O. cuniculus). 
Memorias. Fifth World Rabbit Congress (Francia). C: $1389-1400$.

SÁNCHEZ, S.; RITCHIE, B.; MIZAN, S.; LEE, M. 2000. Pasteurellosis in rabbits. Compendium On Continuing Education For The Practising - Veterinary Learning Systems Co., INC. (EEUU). 22:344351.

SÁNCHEZ, S.; MIZAN, S.; QUIST, C.; SCHRODER, P.; JUNEAU, M.; DAWE, D.; RITCHIE, B.; LEE, M. 2004. Serological response to Pasteurella multocida NanH sialidase in persistently colonized rabbits. Clin. Diagn. Lab. Immunol. (EEUU). 11(5):825-834.

SHIMIZU, T.; TAKAHASHI, Y.; KAWAGUCHI, S.; SAKAKURA, Y. 1996. Hypertrophic and metaplastic changes of goblet cells in rat nasal epithelium induced by endotoxin. Am. J. Resp. Crit Care Med. 153:1412-1418.

SHIMIZU, T.; SHIMIZU, S.; HATTORI, R.; GABAZZA, R.; MAJIMA, R. 2003. A mechanism of antigeninduced goblet cell degranulation in the nasal epithelium of sensitized rats. J Allergy Clin Immunol. (EEUU). 112(1):119-25

SELEIM, R. 1996. Study mayor virulence factors of $P$. multocida isolated from different sources. New Egypt J. Med. 14(6):60-64.

SUCKOW, M.; MARTIN, B.; BOWERSOCK, T.; DOUGLAS, F. 1996. Derivation of Pasteurella multo- cida-free rabbit litters by enrofloxacin treatment. Vet. Microbiol. 51(1-2):161-168.

TAKASHIMA, H.; SAKAI, H.; MASEGI, T. 2001. Detection of antibodies against Pasteurella multocida using immunohistochemical staining in an outbreak of rabbit pasteurellosis. J Vet Med Sci. (Japón). 63(2):171-4.

VENAILLE, T.; MENDI, A.; PHILLIPS, M.; THOMSON, P.; ROBINSON, B. 1995. Role of neutrophils in mediating human apithelial cell detachment from native basement membrane. J. Allergy Clin. Immunol. (EEUU). 95: 597-606.

WAGNER, J.G.; VAN DYKEN, S.J.; WIERENGA, J.R.; HOTCHKISS, J.A.; HARKEMA, J.R. 2003. Ozone exposure enhances endotoxin-induced mucous cell metaplasia in rat pulmonary airways. Toxicol. Sci. (USA). 74(2):437-446.

WATT J.; SWIATLO, E.; WADE, M.; CHAMPLIN, M. 2003. Regulation of capsule biosynthesis in serotype A strains of Pasteurella multocida. FEMS Microbiol. Letters (Inglaterra). 225(1):9-14.

WILLIAMS, A.; TOPLEY, M.; WILLIAMS, B. 1994. Effect of liposomally encapsulated MTX-DMPE conjugates upon TNF alpha and PGE2 release by lipopolysaccharide stimulated rat peritoneal macrophages. Biochim Biophys Acta. (Holanda). $11 ; 1225(2): 217-222$.

Recibido: Febrero 8 de 2007

Aceptado: Abril 17 de 2007 